\title{
Numerical performance of AOR methods in solving first order composite closed Newton-Cotes quadrature algebraic equations
}

\begin{abstract}
In this paper, the application of the Accelerated Over-Relaxation (AOR) iterative method is extended to solve first order composite closed Newton-Cotes quadrature (1-CCNC) algebraic equations arising from second kind linear Fredholm integral equations. The formulation and implementation of the method are also discussed. In addition, numerical results by solving several test problems are included and compared with the conventional iterative methods.
\end{abstract}

Keyword: Accelerated method; Linear Fredholm integral equation; Newton-Cotes quadrature scheme 